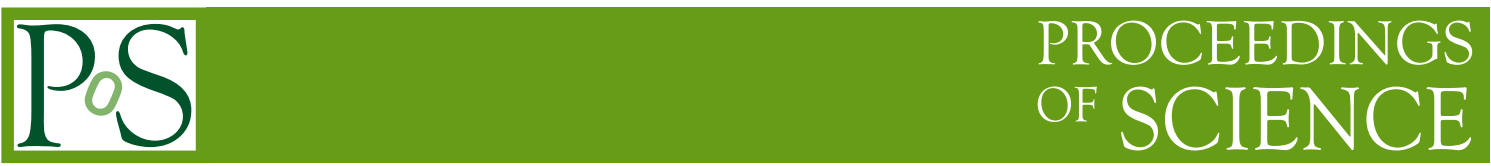

\title{
Diffractive rho and phi production in DIS at HERA
}

\author{
Xavier Janssen*, on behalf of H1 and ZEUS Collaborations. \\ Université Libre de Bruxelles, Belgium. \\ E-mail: xjanssen@ulb.ac.be
}

\begin{abstract}
These proceedings report on $\mathrm{H} 1$ and ZEUS results on diffractive electroproduction of $\rho$ and $\phi$ at the HERA electron-proton collider with data collected between 1996 and 2000. The ZEUS analysis on elastic $\rho$ production, corresponding to an integrated luminosity of $120 \mathrm{pb}^{-1}$, was carried out in the kinematic range of photon virtuality $2<Q^{2}<160 \mathrm{GeV}^{2}$ and $\gamma^{*} p$ centre-of-mass energy $32<W<180 \mathrm{GeV}$. The $\mathrm{H} 1$ collaboration studied $\rho$ and $\phi$ production, both in the elastic and proton dissociative channels, in the kinematic domain $2.5<Q^{2}<60 \mathrm{GeV}^{2}$ and $30<\mathrm{W}<180 \mathrm{GeV}$, with an integrated luminosity of $51 \mathrm{pb}^{-1}$. The total, longitudinal and transverse cross sections as well the s-channel helicity conserving and violating amplitudes are measured as a function of $Q^{2}$, $W$ and $|t|$, the squared-four-momentum transfer to the proton. Emphasis is put on the transition from soft to hard diffraction and spin dynamics. A consistent picture of vector meson production at intermediate and large $Q^{2}$ emerges from the HERA measurements, which can be interpreted in a QCD framework.
\end{abstract}

European Physical Society Europhysics Conference on High Energy Physics, EPS-HEP 2009,

July 16 - 222009

Krakow, Poland

${ }^{*}$ The author is supported by the FRS-FNRS (Belgium). 


\section{Introduction}

These analyses are devoted to the study of diffractive electroproduction of $\rho$ and $\phi$ vector mesons (designed in the following as VM) at HERA, in the elastic and in the proton dissociative channels: $e+p \rightarrow e+Y+V M ; \rho \rightarrow \pi^{+}+\pi^{-}$and $\phi \rightarrow K^{+}+K^{-}$, where $Y$ represents the elastically scattered proton or a diffractively excited baryonic system, well separated in rapidity from the vector meson.

The H1 experiment analysed all four channels [1] with a total luminosity of $51 \mathrm{pb}^{-1}$ in the kinematic domain $2.5<Q^{2}<60 \mathrm{GeV}^{2}, 35<W<180 \mathrm{GeV},|t|<3 \mathrm{GeV}^{2}$ and $M_{Y}<5 \mathrm{GeV} / \mathrm{c}^{2}$, where $Q^{2}=-q^{2}, q$ being the virtual photon four-momentum, $W$ is the photon-proton centre of mass energy and $t$ is the square of the four-momentum transfer from the incident proton to the scattered system $Y$, of which the mass is $M_{Y}$. The ZEUS collaboration concentrated on elastic $\rho$ production [⿰], with a luminosity of $120 \mathrm{pb}^{-1}$, in the domain $2<Q^{2}<160 \mathrm{GeV}^{2}, 32<W<$ $180 \mathrm{GeV}$ and $|t|<1 \mathrm{GeV}^{2}$. The interacting particles were electrons or positrons ${ }^{1}$ of energy 27.5 $\mathrm{GeV}$, colliding with 820 or $920 \mathrm{GeV}$ protons, which corresponds to electron-proton centre of mass energies of $\sqrt{s}=300$ and $320 \mathrm{GeV}$, respectively.
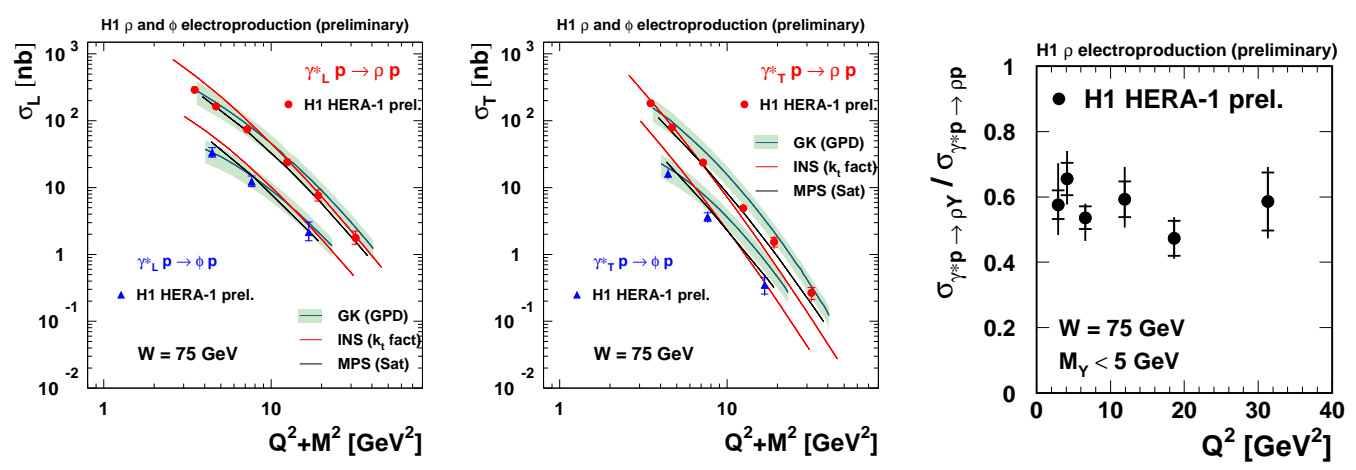

Figure 1: $Q^{2}$ dependency of the (left) longitudinal and (center) transverse $\gamma^{*} \mathrm{p}$ cross sections for elastic $\rho$ and $\phi$ production for $W=75 \mathrm{GeV}$. (right) Ratio as a function of $Q^{2}$ of the elastic and proton dissociative $\gamma^{*} \mathrm{p}$ cross sections for $\rho$ mesons for $W=75 \mathrm{GeV}$ and $M_{Y}<5 \mathrm{GeV} / \mathrm{c}^{2}$.

The $Q^{2}$ range covers the transition region from soft to hard diffraction in which data are interpreted in terms of two complementary QCD approaches. High energy VM production can be described in the proton rest frame as the factorisation of three contributions: the fluctuation of the virtual photon into a $q \bar{q}$ colour dipole, the diffractive dipole-proton scattering, the $q \bar{q}$ recombination into the final state VM. The interaction scale $\mu$ is given by the transverse size of the dipole, $\mu^{2} \simeq\left(Q^{2}+M_{V}^{2}\right) / 4$, for light VM production by longitudinally polarised photons as well as for heavy VM production like $J / \psi$. The scale may however be significantly lower for light VM production by transversely polarised photons following from end-points contributions in the photon wave function. On the other hand, following a QCD factorisation theorem, the longitudinal amplitude $^{2}$ can be described by the convolution of the hard process with generalised parton distributions

\footnotetext{
${ }^{1}$ In the rest of this paper, the word "electron" will be generically used for electron and positron.

${ }^{2}$ Factorisation is proved for longitudinal amplitudes of light VM's and should also apply to heavy VM; its validity is often extended to transverse amplitudes for sufficient $Q^{2}$.
} 
in the proton (GPDs) which describe the probability to find two partons at the same time in the proton at different $x$ values and for given values of $Q^{2}$ and $t$. Several models based on either approach are compared to data; references and brief descriptions can be found in [1, 2].

\section{Cross section measurements}

The $Q^{2}+M^{2}$ dependency of the longitudinal and transverse $\gamma^{*} \mathrm{p}$ cross sections as measured by $\mathrm{H} 1$ are presented in Fig. 11 for elastic $\rho$ and $\phi$ production at $W=75 \mathrm{GeV}$. Overall normalisation uncertainties of $4.7 \%$ for $\rho$ and $5.4 \%$ for $\phi$ mesons are not included in the error bars. The GPD model predictions (blue area) are slightly too hard, both for $\sigma_{L}$ and for $\sigma_{T}$, but the global normalisations are within the theoretical and experimental uncertainties. The dipole $k_{t}$-unintegrated model (red line) gives predictions which are too high and also too steep for $\sigma_{T}$. The dipole saturation model (black line) describes the data rather well, although falling slightly too fast with $Q^{2}+M^{2}$.

Figure 1]c presents, as a function of $Q^{2}$, the ratios of the elastic and proton dissociative $\gamma^{*} \mathrm{p}$ cross sections for $\rho$ mesons for $W=75 \mathrm{GeV}$ and $M_{Y}<5 \mathrm{GeV} / \mathrm{c}^{2}$ as measured by H1. The overall normalisation error on the ratios, which is not included in the error bars, is $2.4 \%$. No significant dependence on $Q^{2}$ of the ratios is observed, supporting the factorisation of diffractive amplitudes into photon vertex contributions and proton vertex processes.
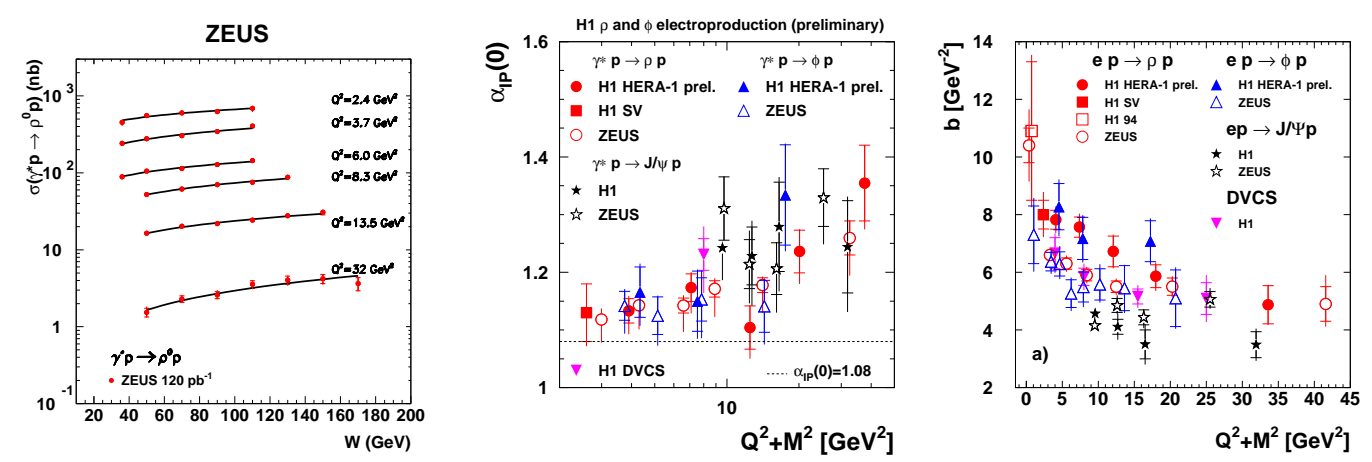

Figure 2: (left) $W$ dependency of the $\gamma^{*} \mathrm{p}$ cross sections for elastic $\rho$ production at several $Q^{2}$ values; Evolution with $Q^{2}+M^{2}$ of the parameters (center) $\alpha_{\mathbb{P}}(0)$ and (right) $b$ for elastic VM production and DVCS.

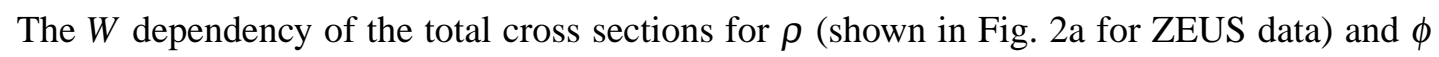
production have been extracted for several $Q^{2}$ values and are characterised by power laws of the form $\sigma \propto W^{\delta}$. This parametrisation is inspired by the Regge description of soft interactions via the exchange of the Pomeron $(\mathbb{P})$ trajectory, with $\delta \simeq 4\left(\alpha_{\mathbb{P}}(\langle t\rangle)-1\right), \alpha_{\mathbb{P}}(t)=\alpha_{\mathbb{P}}(0)+\alpha^{\prime} t$. In soft interactions, typical values for the intercept are $\alpha_{\mathbb{P}}(0) \simeq 1.08-1.11$. The $W$ dependency of $\rho, \phi$ elastic production cross sections are summarised in Fig. $2 \mathrm{~b}$ in the form of the $Q^{2}+M^{2}$ dependence of $\alpha_{\mathbb{P}}(0)$ including earlier measurements and $J / \psi$ data. For light vector mesons, $W$ dependency of the elastic cross section are close or slightly harder than the soft behaviour up to $Q^{2}+M^{2}$ values of the order of $10 \mathrm{GeV}^{2}$. An increase is then observed, up to values of $\alpha_{\mathbb{P}}(0)$ of the order of 1.2-1.3, compatible with $J / \psi$ measurements. This is related to the hardening of the gluon distribution with the scale and confirms a transition from soft to hard diffraction. 
For $|t|<1-2 \mathrm{GeV}^{2}$, the $|t|$ dependency of $\mathrm{VM}$ cross sections are characterised by falling exponential behaviour with slopes $b: d \sigma / d t \propto e^{-b|t|}$. Neglecting the effect of the VM wave function, the $t$ slope is the convolution of the transverse sizes of the interacting object: $b=b_{q \bar{q}}+b_{Y}+b_{I P}$, with contributions from the dipole sizes, of the diffractively scattered system $Y$ (elastic or dissociative proton) and of the exchange ("Pomeron") system. Universal $t$ slopes are thus expected for all VM with the same $q \bar{q}$ dipole sizes, i.e. with same value of the scale $\mu^{2}=\left(Q^{2}+M_{V}^{2}\right) / 4$. Fig. $2 \mathrm{c}$ presents measurements of $b$ slopes for elastic production of several VM and DVCS. For $\rho$ and $\phi$, a strong decrease of the $t$ slopes for increasing $Q^{2}+M_{V}^{2}$ is observed with is attributed to nonperturbative soft contributions from the transverse cross section. For $Q^{2}+M_{V}^{2}>10$ to $20 \mathrm{GeV}^{2}, b$ values similar to those of $J / \psi$ production are reached indicating that the perturbative hard regime starts to be dominant.

\section{Polarisation measurements}

Information on the spin and parity properties of the exchange and on the contributions of the various polarisation amplitudes are accessed through the distributions of production and decay angles which characterise VM production and two-body decays. These angular distributions allow the measurement at HERA of 15 spin density matrix elements (SDME), given in the form $r_{j k}^{i}$, which are normalised bilinear combinations of the complex helicity amplitudes $T_{\lambda_{V}}, \lambda_{\gamma}, \lambda_{\gamma}$ and $\lambda_{V}$ being the helicities of the virtual photon and of the VM.
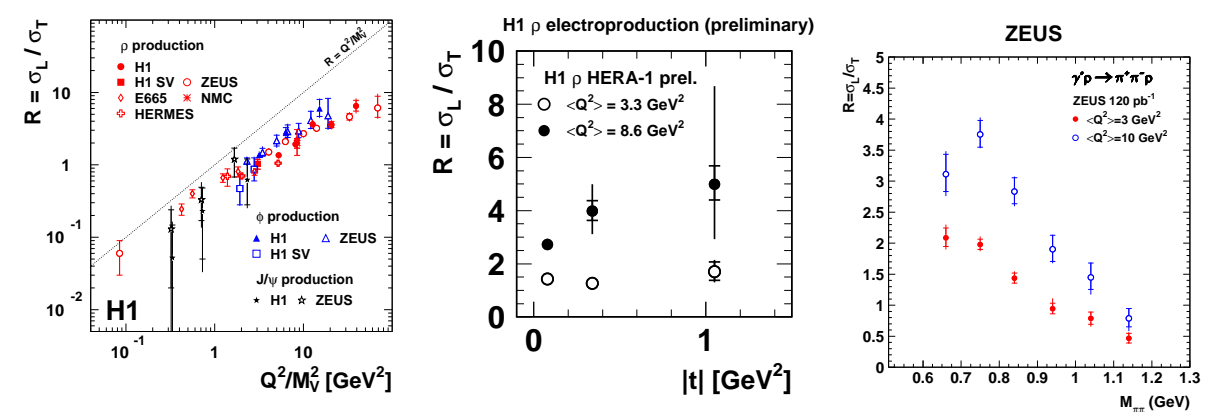

Figure 3: $R=\sigma_{L} / \sigma_{T}$ as a function of (left) $Q^{2} / M_{V}^{2}$ for several VM, (center) $t$ for $\rho$ and (right) $m_{\pi \pi}$ for $\rho$ productions.

H1 and ZEUS have measured for $\rho$ and $\phi$ mesons production the $Q^{2}, W, t$ and (for $\rho$ only) the invariant mass $m_{\pi \pi}$ dependency of these 15 SDME's from which the ratio between the longitudinal $\left(\sigma_{L}\right)$ and the transverse $\left(\sigma_{T}\right) \gamma^{*}$ p cross-sections can be extracted. Fig. 3 shows the ratio $R=\sigma_{L} / \sigma_{T}$ as a function of $Q^{2} / M_{V}^{2}$ for several VM and as a function of $t$ and the two pions invariant mass $m_{\pi \pi}$ for $\rho$ meson production. An universal behaviour for all $\mathrm{VM}$ is observed as a function of $Q^{2} / M_{V}^{2}$ as expected from the LO naive perturbative QCD predictions. However, deviations from the linear dependence reflects the gluon dynamics and higher order effects. An increase of $R$ with $|t|$ is observed at high $Q^{2}$. This can be translated into a measurement of the difference between the longitudinal and transverse $t$ slopes $b_{L}$ and $b_{T}: R(t) \propto \exp \left(-\left(b_{L}-b_{T}\right)|t|\right)$. A slight indication $(1.5 \sigma)$ is found for a negative value of $b_{L}-b_{T}$ suggesting that, as expected, the average transverse 
size of dipoles for transverse amplitudes is larger than for longitudinal amplitudes. A striking decrease of the cross section ratio $R$ with the increasing $m_{\pi \pi}$ is observed. A simple interpretation of the $m_{\pi \pi}$ dependence follows from the general, "naive" $Q^{2} / M^{2}$ dependence of the cross section ratio, if the mass $M$ is understood as that of the quark pair (i.e. the dipion mass), rather than the nominal resonance mass.
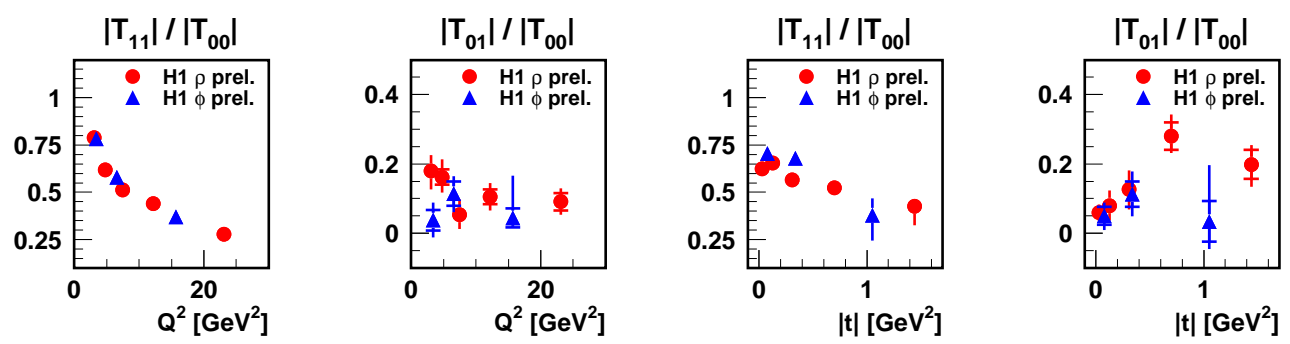

Figure 4: Amplitude ratios as a function of $Q^{2}$ and $|t|$ for $\rho$ and $\phi$ productions.

Following the presentation in ref. [3], the amplitude relative strengths, measured with reference to the dominant $T_{00}$ amplitude, are computed from global fits to the 15 SDME measurements, all amplitudes being taken as purely imaginary. Results are shown in Fig. 7 as a function of $Q^{2}$ and $|t|$ both for $\rho$ and $\phi$ production. The strong decrease with $Q^{2}$ of the $\left|T_{11}\right| /\left|T_{00}\right|$ amplitude ratio is related to the usual $Q^{2}$ dependence of the cross section ratio $R=\sigma_{L} / \sigma_{T}$. For the first time, a $Q^{2}$ dependence of the non $s$-channel helicity conservation (SCHC) $\left|T_{01}\right| /\left|T_{00}\right|$ ratio is observed. The normalised $T_{01}$ helicity flip amplitude is increasing with $|t|$ confirming previous SCHC violation observation. Conversely, and for the first time, a decrease with $|t|$ of the $\left|T_{11}\right| /\left|T_{00}\right|$ amplitude ratio, for $\rho$ and for $\phi$ production is observed. The decrease of $\left|T_{11}\right| /\left|T_{00}\right|$ with $|t|$, which is not taken into account in perturbative QCD calculations [3], is needed to compensates the increase of $\left|T_{01}\right| /\left|T_{00}\right|$ and ensure unitarity.

\section{Conclusions}

This paper reports on the measurement $\rho$ and $\phi$ vector meson production at high $Q^{2}$. The hardening of the $W$ dependence and the decrease of the $b$ slopes at high $Q^{2}$ indicate a transition from soft to hard diffraction. Proton vertex factorisation is supported by the constancy with $Q^{2}$ of the ratio of the proton dissociative to elastic cross sections. Ratio of polarised amplitudes have been extracted confirming SCHC violation.

\section{References}

[1] F.D. Aaron et al. [H1 Collaboration], DESY-09-093, to be submitted to JHEP. H1prelim-08-013 and H1prelim-09-017 available from http://www-h1.desy.de.

[2] S. Chekanov et al. [ZEUS Collaboration], PMC Physics A 1, 6 [hep-ex/0708.1478].

[3] D.Yu. Ivanov and R. Kirschner, Phys. Rev. D58 (1998) 114026 [hep-ph/9807324]. 